\title{
Enzyme-linked immunosorbent assay (ELISA) for the detection of antibodies to Angiostrongylus cantonensis
}

\author{
S. N. CHEN \\ Dept. of Zoology, National Taiwan University, Taipei, Taiwan, Republic of China
}

\begin{abstract}
The enzyme-linked immunosorbent assay (ELISA) was employed for the sero-diagnosis of Angiostrongylus cantonensis infections in rats and man. Metabolic and 'purified' antigens of adult or juvenile $A$. cantonensis were evaluated by ELISA and compared against Toxocara canis antigen for sensitivity and specificity. Sera and cerebrospinal fluid (CSF) from both rats and man gave higher ELISA values against antigens of $A$. cantonensis than those obtained from negative control samples. The results showed that cross reaction occurred between $T$. canis and $A$. cantonensis. Of four $A$. cantonensis antigens tested, a Sephacryl S-300 "purified' fraction was shown to be superior in the ELISA to determine angiostrongyliasis.
\end{abstract}

\section{Introduction}

Angiostrongylus cantonensis (Chen, 1933) is a causative agent of human eosinophilic meningitis in Southeast Asia and the Pacific Islands. The characteristic symptoms of patients with this disease are often confused with other central nervous disorders and as a result few cases of angiostrongyliasis would be diagnosed. Proven cases of human infection showing worms from cerebrospinal fluid (CSF) are rare (CHEN, 1979) and a reliable diagnostic assay, specific for angiostrongyliasis, is urgently needed to confirm diagnosis.

Recently, ThaRavanI, (1979) reported that ELISA using an $A$. cantonensis antigen prepared from larvae recovered from rat brain, was a most promising technique for the sero-diagnosis of angiostrongyliasis. WELCH et al. (1980) reported the successful development of both serum and cellular immunity assays to determine infection with $A$. cantonensis in animal and human hosts.

This study is an assessment of the sensitivity and specificity of ELISA in the diagnosis of $A$. cantonensis infections in rat and man by using crude and purified antigens prepared from adult and juvenile $A$. cantonensis.

\footnotetext{
Materials and Methods

\section{Antigen preparation}

Infective larvae of $A$. cantonensis were collected as described previously (CHEN et al., 1981). Albino rats were orally infected with the larvae using a small rubber tube. Juvenile and adult worms of $A$. cantonenis were obtained from the subarachnoid space of brain and pulmonary artery of rats at 22 or 80 days after infection, respeetively. Adult $T$. canis were collected from intestines of infected dogs.

Motile $A$. cantonensis and $T$. canis were washed three times in normal saline and then homogenized in $0.05 \mathrm{M}$ carbonic buffer solution (pH 9.6) using a glass microhomogenizer (Belco, Vineland, New Jersey, USA). Subsequently, the homogenized materials were centrifuged at $10,000 \mathrm{~g}\left(4^{\circ} \mathrm{C}\right)$ for $30 \mathrm{~min}$. The supernatants were measured for the protein concentration by utilizing Folin reagent as described by LOWRY et al. (1951) and stored at $-70^{\circ} \mathrm{C}$.

Washed, live adult $A$. cantonensis were incubated in a medium containing normal saline plus $0.5 \%$ glucose at $37^{\circ} \mathrm{C}$ for 24 hours for the preparation of metabolic antigen. The culture medium was collected and the protein concentration in the medium was measured as described above.
}

\section{Gel filtration}

The crude antigen of adult $A$. cantonensis was fractionated on Sephacryl S-300. The gel was equilibrated with $0.05 \mathrm{M}$ carbonic buffer, $\mathrm{pH} 9.6$ and packed in a $2.6 \times 36 \mathrm{~cm}$ column at $4^{\circ} \mathrm{C}$. The antigen containing approximately $20 \mathrm{mg}$ of total protein was mixed with $2 \mathrm{ml}$ of $0.05 \mathrm{M}$ carbonic buffer, applied to the column and eluted with the same buffered solution. Fractions of $2 \mathrm{ml} /$ tube were collected at a flow rate of $11 \mathrm{ml} \mathrm{cm} \mathrm{ch}^{-2}$. These were monitored photometrically at $280 \mathrm{~nm}$ by using a Gilford $250 \mathrm{Spec}-$ trophotometer. Each fraction was then used as an antigen in the ELISA.

Selected fractions were collected, concentrated using celluphane tubes and dialysed in $0.05 \mathrm{M}$ carbonic buffer $(\mathrm{pH}$ $9 \cdot 6$ ). Protein concentrations were then measured on each fraction, called 'purified' antigen. These purified antigens were used in subsequent experiments.

\section{Antisera}

Sera from rats were obtained before, and then at weekly intervals, after oral infection (eight albino rats each infected with 100 third-stage larvae). Positive human sera and CSF were obtained from three patients with parasitologically proven $A$. cantonensis in the CSF. Sera and CSF from five confirmed Japanese encephalitis patients were employed as negative controls. The samples were obtained from the National Taiwan University Hospital.

Enzyme-linked Immunosorbent Assay (ELISA)

ELISA was performed in polystyrene EIA microtitration plates containing 96 flat-bottomed wells (Linbro, Flow Laboratories, Inc., McLearn, Virginia, USA). The procedures used were modified after thuse described by VOLLER $t$ al. (1974).

The optimal serum dilution was found to be $1: 50$ in phosphate buffered saline (PBS). Carbonic buffer ( $\mathrm{pH} 9 \cdot 6$ ) was also suitable for the coating procedure and this system was employed for the detection of antibodies from infected rat and human sera and CSF.

For coating procedures, $0.1 \mathrm{ml}$ of each crude antigen with a total protein concentration of $25 \mu \mathrm{g} / \mathrm{ml}$ was added to each well. The plates were then sealed, incubated at $4^{\circ} \mathrm{C}$ overnight and washed three times with PBS containing 0.05\% Tween 20 (PBST). Serial dilutions of test sera were then added immediately to the wells. The plates were incubated at $37^{\circ} \mathrm{C}$ for three hours and then washed with PBST. After shaking dry, $0.1 \mathrm{ml}$ peroxidase-labelled rabbit anti-human $(1: 200$ dilution) or anti-rat immunoglobulin conjugate in PBS (1:50 dilution) was added and incubated for four hours at $37^{\circ} \mathrm{C}$. Anti-human and anti-rat rabbit sera were purchased from US Biochemical Corporation and the substrate from Sigma Chemical Co., St. Louis, Missouri, USA. Excess conjugate 
was removed by washing five times with PBST. Bovine serum albumin (BSA, Sigma Chemical Co., St. Louis, Missouri, USA) was used as a control.

The detection of antibody against $A$. cantonensis in human sera and CSF was done by coating each well with $0.1 \mathrm{ml}$ of 'purified' antigen at a protein concentration of $25 \mu \mathrm{g} / \mathrm{ml}$. Fresh substrate solution was prepared by dissolving $10 \mathrm{mg}$ of 0 -phenylenediamine in $0.1 \mathrm{M}$ PBS containing $1 \mu \mathrm{l} 30 \%$ $\mathrm{H}_{2} \mathrm{O}_{2}$ and then added into each well. After incubation at room temperature for $50 \mathrm{~min}$, the colour reaction was stopped by the addition of $0.05 \mathrm{ml} 1 \mathrm{~N} \mathrm{NaOH}$. The absorbance in each well was read at $450 \mathrm{~nm}$ using a Titertek Multiskan (Flow Laboratories).

Values are expressed as two ratios (VOLLER et al., 1979 and YOLKEN et al., 1977) calculated from ELISA readings for both tested and referenced sera.

\section{Results \\ Optimal buffered solutions for antigens and antibodies}

EIA microplates were incubated with adult $A$. cantonensis antigen diluted in carbonic buffer and carbonic buffer plus $1 \% \mathrm{BSA}$, then reacted with anti-serum in various buffered solution including PBS, PBST and PBST $+1 \%$ BSA. The results of ELISA showed that there were no significant differences in coating efficiency and in the reactions between antigen and antibody by using different buffered solutions or supplementations. Therefore PBS and carbonic buffer were used for all further experiments.

\section{Animal experiments}

Serum from eight laboratory-infected rats showed that antibudies to $A$. cantonensis could be demons- trated from three weeks to eight weeks following the ingestion of the third-stage larvae. The sera from all infected animals showed ELISA values that increased with time after infection. Significant decrease in ELISA value was observed with the increase of serial dilutions ranged from $2^{-2}$ to $2^{-11}$.

Significantly higher ELISA values were detected in sera from infected rats (three weeks and up to 26 weeks after infection) than in non-infected control rat sera (Figs. 1 and 2).

Of the three $A$. cantonensis crude antigens used, the highest ELISA values were observed with the adult worm antigen. High ELISA values were determined with $A$. cantonensis positive sera against $T$. canis antigen which suggested considerable cross reaction between these two nematodes (Fig. 2).

Table I gives the ratios calculated from the ELISA values obtained from sera of normal and infected animals. The results show that positive sera gave higher ratios than normal sera. In addition these ratios indicated that adult worm antigen was superior to the other crude antigens.

The results obtained after fractionation of adult worm antigen using Sephacryl S-300 showed five protein peaks detected at $280 \mathrm{~nm}$. When individual fractions of $2 \mathrm{ml}$ were tested against the infected rat sera, the higher ELISA value was obtained in the second high molecular weight. In comparison, low ELISA values were obtained with the same fraction reacted with the normal rat sera. The fractions with lower molecular weight revealed little specificity against $A$. cantonensis infected rat sera (Fig. 3).

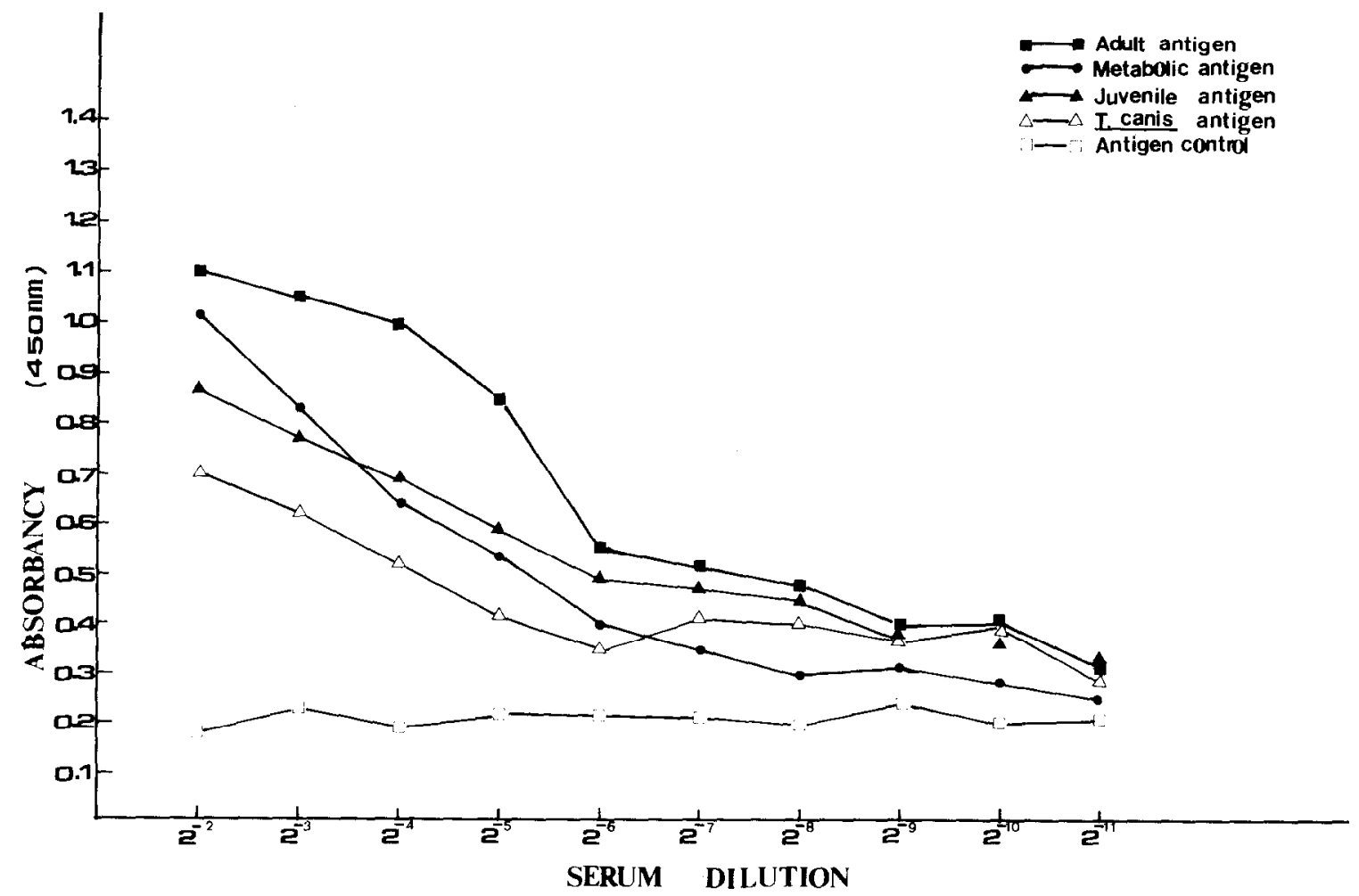

Fig. 1. The ELISA values of sera obtained from 8 rats infected with 100 Angiostrongylus cantonensis infective larvae at 8 weeks after infection by using adult worm, juvenile worm or metabolic antigen of Angiostrongylus cantonensis and Toxocara canis antigen. 


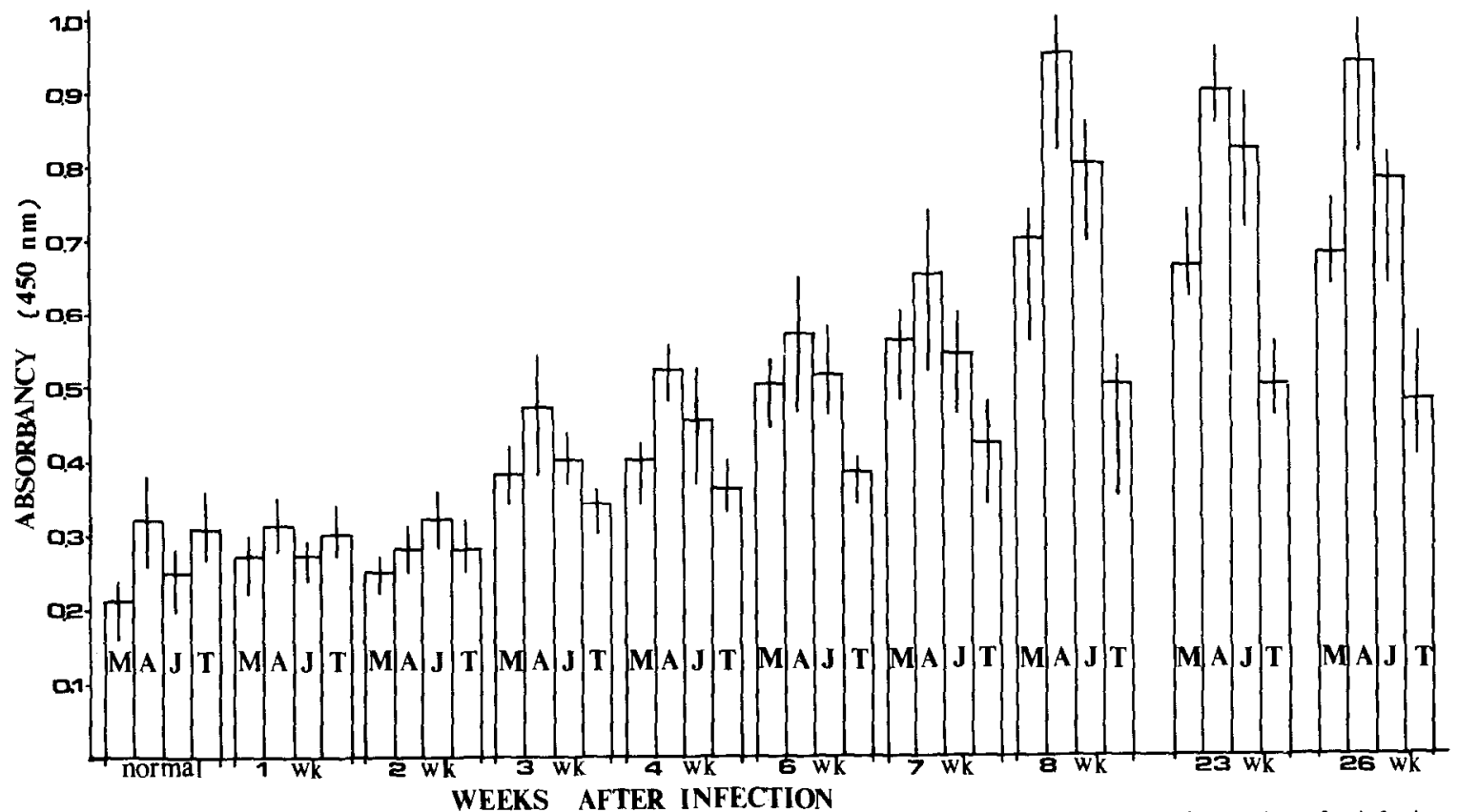

Fig. 2. The ELISA values of sera obtained from 8 rats infected with 100 Angiostrongylus cantonensis infective larvae at various periods after infection by using adult worm, juvenile worm or metabolic antigen of Angiostrongylus cantonensis and Toxocara canis antigen. M: Metabolic antigen of $A$. cantomensis A: Adult worm antigen of $A$. cantonensis $\mathrm{J}$ : Juvenile worm antigen of $A$. cantonensis $\mathrm{T}$ : Adult worm antigen of Toxocara canis.

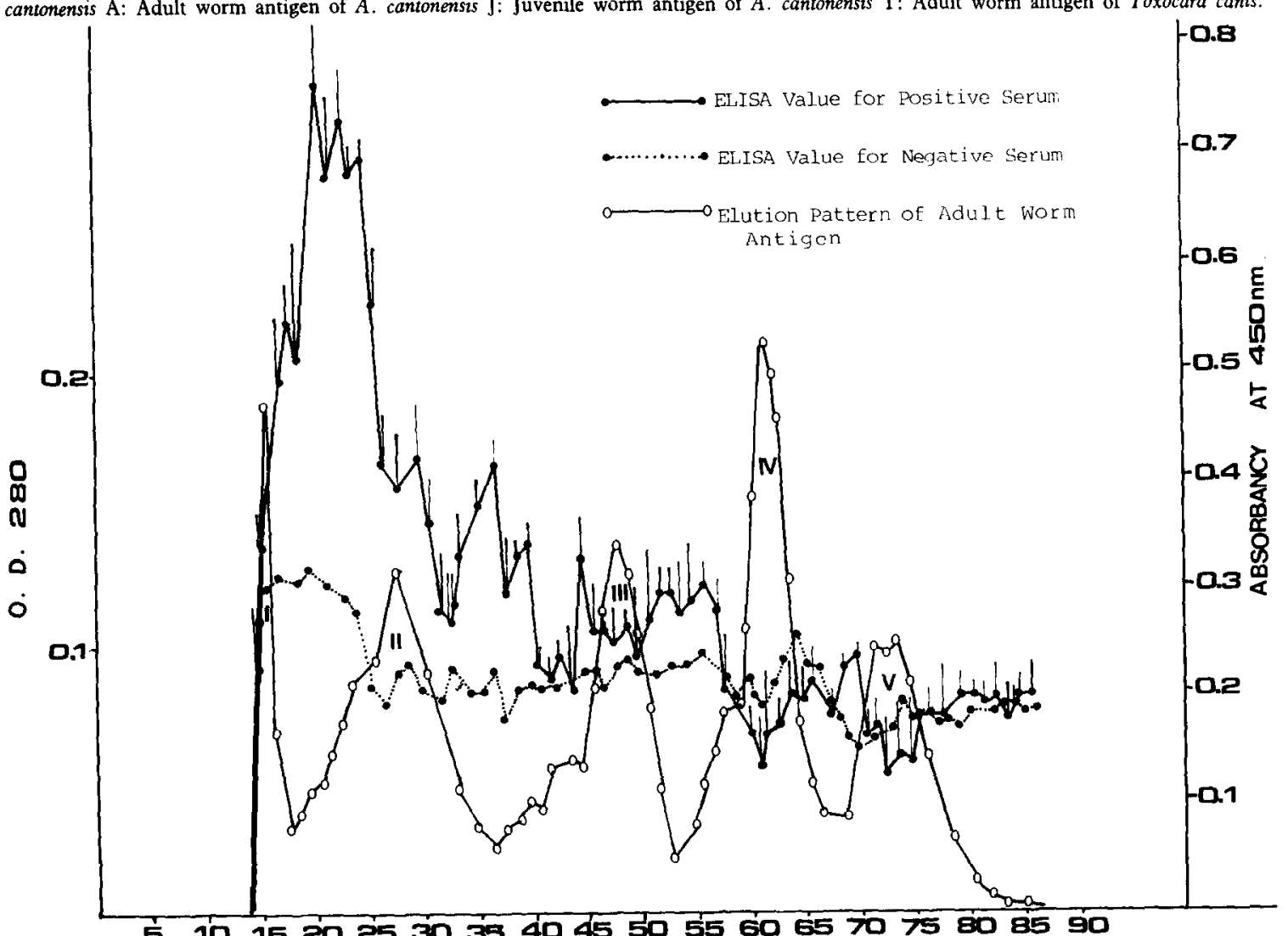

5101520 25 30394045505560657075808590

FRACTION NO.

Fig. 3. The gel filtration of adult worm antigen of Angiostrongylus cantonensis on Sephacryl S-300 column $(2.6 \times 36 \mathrm{~cm}$, in carbonic buffer, $\mathrm{pH} 9 \cdot 6)$ and the ELISA value for each eluate against normal and infected rat sera. The eluates were collected at a volume of $2 \mathrm{ml}$ per tube in a flow rate of $11 \mathrm{ml} \mathrm{cm}^{-2} \mathrm{hr}^{-1}$ and monitored at $280 \mathrm{~nm}$. Each ELISA value was obtained from 8 rats before and after infection of 100 Angiostrongylus cantonensis inferrive laruas Farth eluate was coated in microdlate without measuring the protein concentration. 


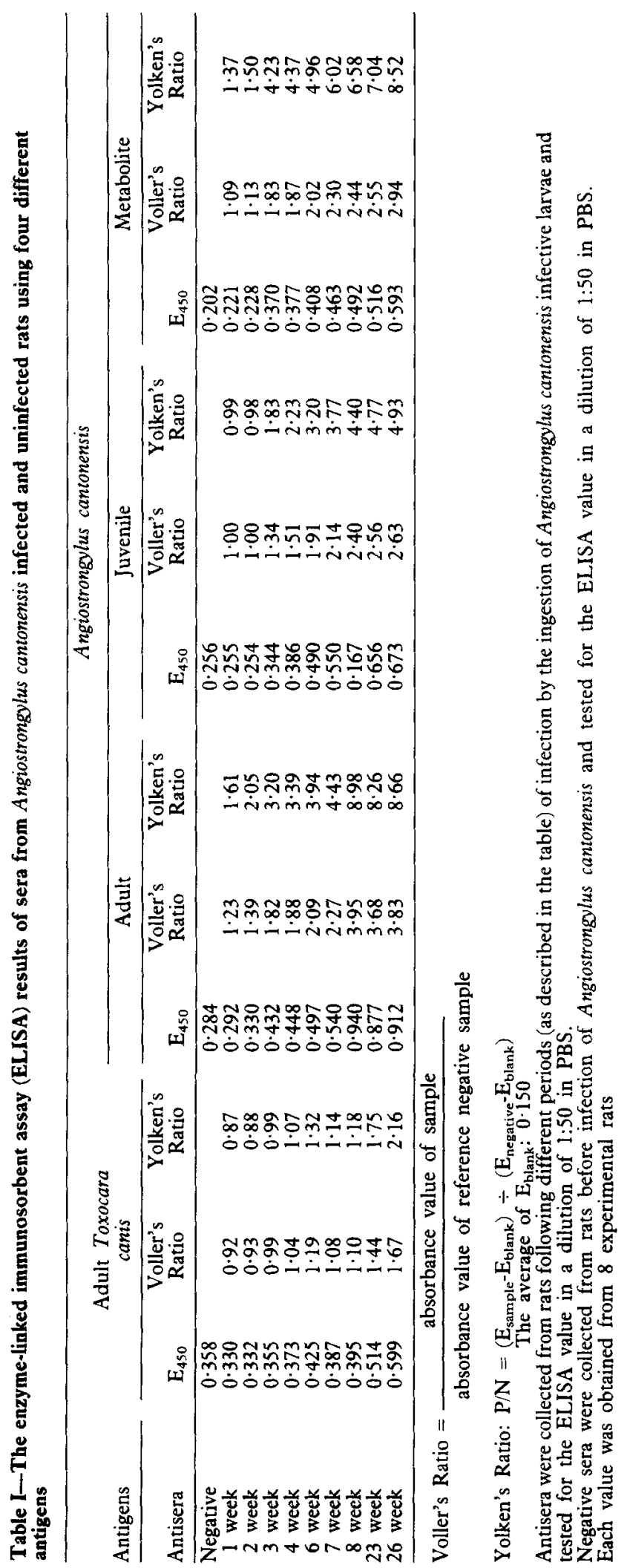




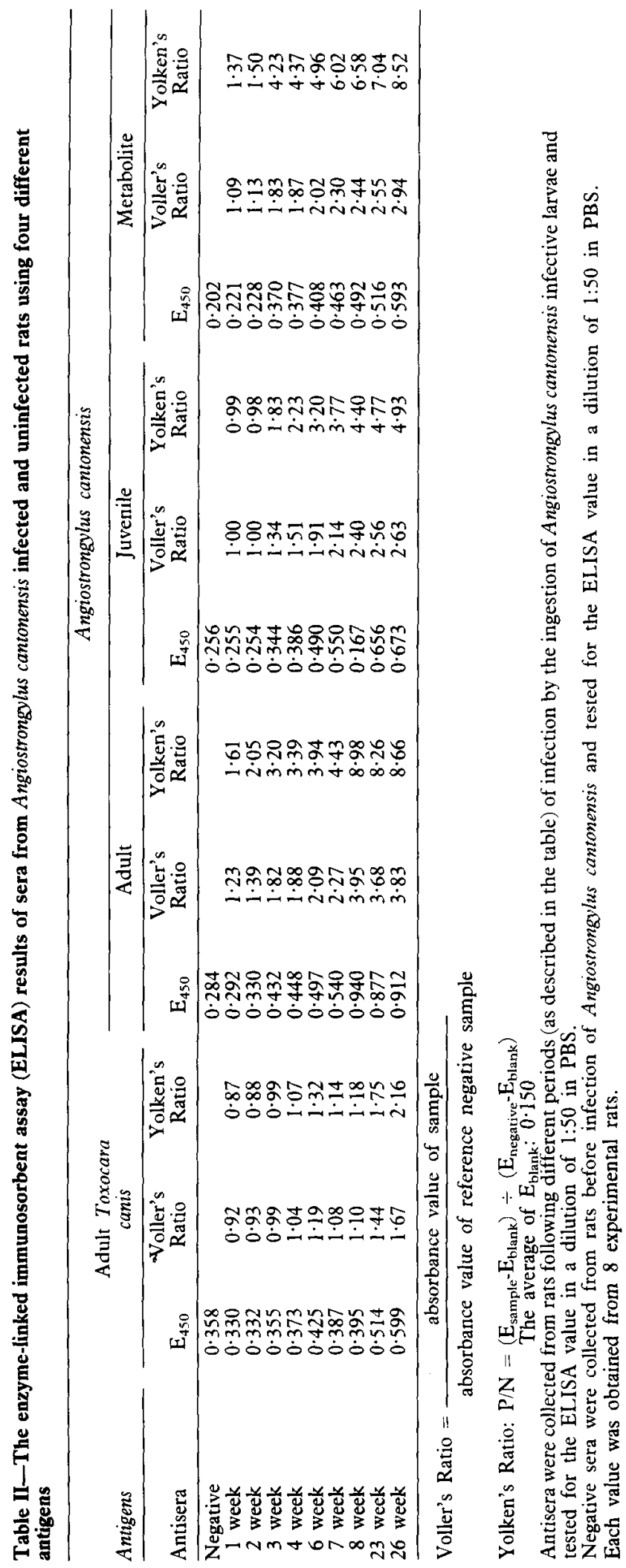




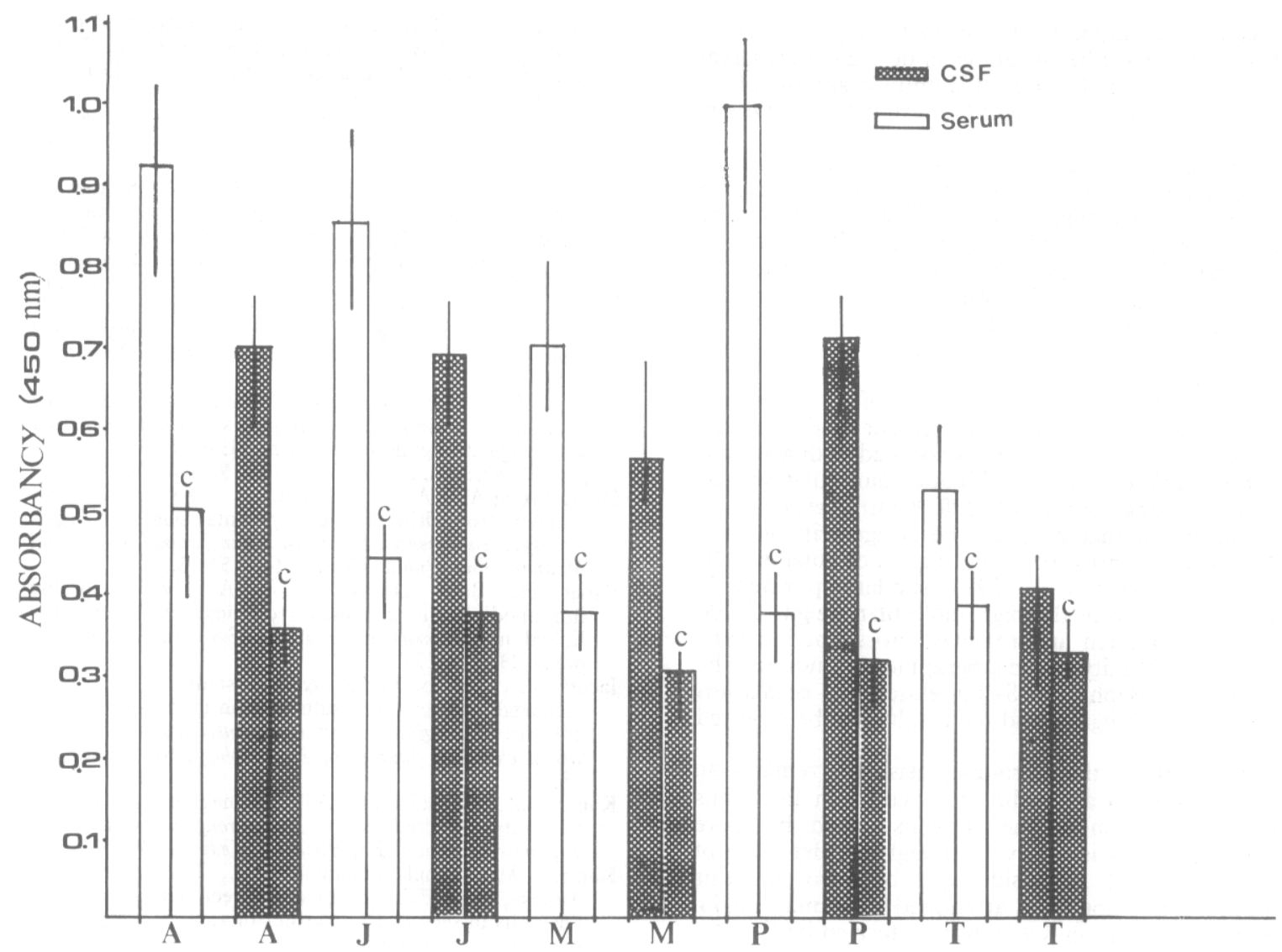

Fig. 4. The ELISA values of normal and Angiostrongylus cantonensis infected sera and cerebrospinal fluids, determined using adult worm, juvenile worm, metabolic or 'purified' antigen of $A$. cantonensis and adult Toxocara canis antigen. The 'purified' antigen was obtained by the filtration on Sephacryl S-300 using carbonic butfer, $\mathrm{pH} 9 \cdot 6$ and coated on the microplate at a total protein concentration of $25 \mu \mathrm{g}$ per well. A: adult worm antigen of $A$. cantonensis, J: Juvenile worm antigen of $A$. cantonensis, $M$ : Metabolic antigen of $A$. cantonensis, P: 'purified' antigen of $A$. cantonensis, $T$ : Adult $T$. canis antigen, C: Negative control. The values were obtained from 3 proven cases of $A$. cantonensis infection and 5 normal individuals.

\section{Human sera and cerebrospinal fluid (CSF)}

The ELISA values of sera and CSF from three $A$. cantonensis infected patients at 1:50 dilution were compared with those obtained from five control individuals. Five antigen preparations $(A$. cantonensis; adult and juvenile crude worm antigen, a metabolic antigen and a purified antigen, and $T$. canis crude worm antigen) were employed in this study. The sera exhibited higher ELISA values than the corresponding CSF sample from each individual tested. The highest values recorded for both sera and CSF were obtained with 'purified' antigen. However, the values obtained using 'purified' antigen against control sera were lower than those obtained with adult or juvenile antigen (Fig. 4). The patients with angiostrongyliasis also gave higher ELISA values (CSF and serum) against $T$. canis antigen than the negative controls.

\section{Discussion}

In studies of laboratory diagnosis of eosinophilic meningitis associated with angiostrongyliasis, CROSS (1978) reported that in all parasitologically confirmed and presumptive cases, ELISA determinations on sera from these individuals were significantly higher than for the control sera. Similarly, in the present study positive and negative sera and CSF of man and experimentally infected rats showed clcar differences in ELISA value. These results confirmed that ELISA can be used to detect and measure antibody in the determination of $A$. cantonensis infections.

In comparison with juvenile worm antigen of $A$. cantonensis, adult worm antigen exhibited a better sensitivity for detection of antibodies in the infected cases. The higher ELISA values in infected rat sera were maintained up to 26 weeks after infection. This suggested that antigenic stimulation was mainly a result of material released by adult worms. A similar suggestion was made by KAMIYA \& TANAKA (1969) who found that the appearance of detectable antibody coincided with appearance of larvae in the faeces. Other workers (JACOBS et al., 1965; KAMIYA et al., 1972) demonstrated that antigenic factors are associated with the adult female, but not adult male, worms. using polyacrylamide gel and radioimmunoprecipitation techniques DHARMKRONG-AT \& SIRI SINHA (1983) demonstrated that polypeptides with molecular weights of $80,000,39,500$ and 22,000 were present in more than one developmental stage and were antigenically related. The 15,500 dalton protein was present primarily in L3s and stimulated a 
considerable amount of antibody response. It is therefore the studies of antigens derived from larval stages on the application of immunodiagnosis of $A$. cantonensis infection in man that are probably important.

The present investigation showed that Sephacryl S-300 'purified' antigen was superior to crude antigen in ELISA determination for angiostrongyliasis. The need for highly purified antigens for ELISA diagnosis of helminthic infections has been demonstrated by other investigators for hydatid disease, onchocerciasis and schistosomiasis in man (FARAG et al., 1975; BARTLETT et al., 1975; BARAKAT et al., 1983; VOLLER et $a l .$, 1976).

The purification of adult antigen of $A$. cantonensis using Sephacryl S-300 in the present study showed that the specific antigen was associated with a second elution peak which was of higher molecular weight fraction. Using Sephadex G-200, KAMIYA et al. (1973) demonstrated that most of the antigenicity for the IHA test was present in the high molecular weight fraction. WELCH et al. (1983) similarly purified $T$. canis antigen from the high molecular weight G-200 Sephadex fraction and removed cross-reacting antigens using affinity chromatographic techniques. The analysis of Sephacryl S-300 eluted $A$. cantonensis antigen is in progress and the results will be reported elsewhere.

It is realized that sero-diagnosis only remains an indirect way to assay the presence of an infectious agent. Detection of the antigens would be more practical and satisfactory. Although the detection of antigens in CSF is considered to be important, this approach has not been attempted in human angiostrongyliasis. The only trial was performed by CHEN $e t$ al. (1972) who used IHA in the experimental monkeys. Unfortunately, the antigen titres recorded in CSF were relatively low and the results obtained from four experimental animals were variable. Monoclonal antibody or specific rabbit antiserum against $A$. cantonensis may also contribute to the discovery of antigens in infected animals.

A reliable technique is needed for the direct and early diagnosis of angiostrongyliasis. The versatility, sensitivity and simplicity of ELISA and its suitability for use in assay to detect circulating antigen suggests the possibility of its application in the detection of antigens in blood or CSF of patients infected by $A$. cantonensis.

\section{Acknowledgements}

This work was sponsored by grant from the National Science Council of the Republic of China (NSC 72-0201. B001A-39).

\section{References}

Barakat, R. M., El-Gassim, E. E., H. N. Awadalla, El-Molla, A. \& Omer, E. A. (1983). Evaluation of enzyme-linked immunosorbent assay (ELISA) as a diagnostic tool for schistosomiasis. Transactions of the Royal Society of Tropical Medicine and Hygiene, 77, 109-111.

Bartlett, A., Bidwell, D. E. \& Voller, A. (1975). Primary studies on the application of enzyme immuno-assay in the detection of antibodies in onchocerciasis. Tropenmedizin und Parasitologie, 26, 370-374.

Chen, E. R. (1979). Angiostrongyliasis and eosinophilic meningitis on Taiwan: a review. In: Studies on Angiostrongyliasis in Eastern Asia and Australia, Cross, J. H. (Editor). Special Publication of the U.S. Medical Research Unit No. 2, Taipei, Taiwan, pp. 57-73.

Chen, H. T. (1933). A preliminary report on a survey of animal parasites of Canton, China rat. Lingnan Science Joumal, 12, 66-74.

Chen, S. N., Suzuki, T. \& Liu, K. H. (1972). Studies on immunodiagnosis of angiostrongyliasis 1. detection of antigen and antibody in serum and cerebrospinal fluid. Foumal of the Formosan Medical Association, 72, 161-166.

Chen, S. N., Tang, T. \& Lee, S. J. (1981). The in vitro cultivation of the first and third stage larvae and adult worm of Angiostrongylus cantonensis. Proceeding of the National Science Council, Republic of China, Part B, 5, 375-384.

Cross, J. H. (1978). Clinical manifestations and laboratory diagnosis of eosinophilic meningitis syndrome associated with angiostrongyliasis. Southeast Asian Journal of Tropical Medicine and Public Health, 9, 161-170.

Dharmkrong-AT, A. \& Sirisinha, S. (1983). Analysis of antigens from different developmental stages of Angiostrongylus cantonensis. Southeast Asian Fournal of Tropical Medicine and Public Health, 14, 154-162.

Farag, H., Bout, D. \& Capron, A. (1975). Specific immunodiagnosis of human hydatidosis by the enzymelinked immunosorbent assay ELISA. Biomedicine Express, 23, 276-279.

Jacobs, L., Lunde, M. N. \& Weinstein, P. P. (1965). Hemagglutination test results with antigens derived from cultures of Angiostrongylus cantonensis and with whole worm extracts. Foumal of Parasitology, 51 (section 2), 38.

Kamiya, M. \& Tanaka, H. (1969). Hemagglutination test in the rats infected with Angiostrongylus cantonensis. Fapanese Fournal of Experimental Medicine, 39, 593-599.

Kamiya, M., Kongkamnuankarn, K., Tharavanij, S. \& Tanaka, H. (1972). Change of indirect hemagglutination reactions in serum after transfer of adult Angiostrongylus cantonensis to abdominal cavity of rats. Southeast Asian Journal of Tropical Medicine and Public Health, 3, 119-123.

Kamiya, M., Tharavanii, S. \& Harinasuta, C. (1973). Antigenicity for hemagglutination and immunoelectrophoresis tests in fractionated antigens form Angiostrongylus cantonensis. Southeast Asian Fournal of Tropical Medicine and Public Health, 4, 187-194.

Lowry, O. H., Rosebrough, Farr, A. C. \& Randall, R. J. (1951). Protein measurement with the Folin phenol reagent. Fournal of Biological Chemistry, 193, 265-275.

Tharavanii, S. (1979). Immunology of angiostrongyliasis. In: Studies on Angiostrongyliasis in Eastern Asia and Australia. Cross, J. H. (Editor), Special Publication of the U.S. Naval Medical Research Unit No. 2, Taipei, Taiwan, pp. 151-164.

Voller, A., Bidwell, D., Huldt, D. \& Engvall, E. (1974). A microplate method of enzyme-linked immunosorbent assay and its application to malaria. Bulletin of the World Health Organization, 51, 209-211.

Voller, A., Bidwell, D. E., Huldt, G. \& Engvall, E. (1976). The enzyme immunoassays in diagnostic medicine. Bulletin of the World Health Organization, 53, 56-65.

Voller, A., Bidwell, D. E. \& Bartlett (1979). The enzyme linked immunosorbent assay (ELISA). A guide with abstracts of microplate applications. Dynatech Europe, Borough House, Rue du Pré, Guernsey, U.K., pp. 31-32.

Welch, J. S., Dobson, C. \& Campbell, G. R. (1980). Immunodiagnosis and seroepidemiology of Angiostrongylus cantonensis zoonoses in man. Transactions of the Royal Society of Tropical Medicine and Hygiene, 74, 614-623.

Welch, J. S., Symons, M. H. \& Dobson, C. (1983). Immunodiagnosis of parasitic zonnoses: purification of Toxocara canis antigens by affinity chromatography. International Fournal for Parasitology, 13, 171-178. 
Yolken, R. H., Kim, H. W., Clem, T., Wyatt, R. G., Kalica, A. R., Chanock, R. M. \& Zapikian, A. Z. (1977). The enzyme-linked immunosorbent assay (ELISA) for the detection of human reovirus-like agent of infantile gastro-enteritis (HRVLA) in human stool Lancet, ii, 363-366.

Accepted for publication 18th March, 1985.

\section{HEALTH AND HEALTH SERVICES \\ FOR \\ PLANTATION WORKERS}

A meeting for health professionals, labour and union officials, development planners and aid officials to consider case studies from Africa and Asia and to discuss future policy implications.

Place: Manson Lecture Theatre,

London School of Hygiene and Tropical Medicine

Keppel St., London WC1E 7HT

Date: Thursday 4th September 1986

Time: 10 a.m.-5 p.m.

There will be no fee for admission. 